\title{
SOLVABILITY FOR A NONLINEAR THREE-POINT BOUNDARY VALUE PROBLEM WITH $p$-LAPLACIAN-LIKE OPERATOR AT RESONANCE
}

\author{
M. GARCÍA-HUIDOBRO, C. P. GUPTA, AND R. MANÁSEVICH
}

Received 20 April 2001

\section{Introduction}

Let $\phi$ be an odd increasing homeomorphism from $\mathbb{R}$ onto $\mathbb{R}$ which satisfies $\phi(0)=0$ and let $f:[a, b] \times \mathbb{R} \times \mathbb{R} \mapsto \mathbb{R}$ be a function satisfying Carathéodory conditions.

Separated two-point and periodic boundary value problems containing the nonlinear operator $\left(\phi\left(u^{\prime}\right)\right)^{\prime}$, or its more particular form, the so-called $p$-Laplace operator, have received a lot of attention lately (cf. $[6,7,8,14,15]$ and the references therein).

On the other hand, three-point (or $m$-point) boundary value problems for the case when $\left(\phi\left(u^{\prime}\right)\right)^{\prime}=u^{\prime \prime}$, that is, the linear operator, have been considered by many authors (cf. [3, 9, 10, 12, 13]).

The purpose of this paper is to study the following three-point boundary value problem which contains the nonlinear operator $\left(\phi\left(u^{\prime}\right)\right)^{\prime}$,

$$
\begin{gathered}
\left(\phi\left(u^{\prime}\right)\right)^{\prime}=f\left(t, u, u^{\prime}\right), \\
u^{\prime}(a)=0, \quad u(\eta)=u(b),
\end{gathered}
$$

where $\eta \in(a, b)$ is given. We are interested in the case when problem (1.1) is at resonance, meaning by this that the associated three-point boundary value problem

$$
\begin{aligned}
& \left(\phi\left(u^{\prime}(t)\right)\right)^{\prime}=0 \quad a<t<b, \\
& u^{\prime}(a)=0, \quad u(\eta)=u(b)
\end{aligned}
$$

has the nontrivial solution $u(t)=A$, where $A \in \mathbb{R}$ is an arbitrary constant. For the linear operator, three-point boundary value problems at resonance have been recently studied in $[3,11]$.

Copyright (C) 2001 Hindawi Publishing Corporation Abstract and Applied Analysis 6:4 (2001) 191-213 2000 Mathematics Subject Classification: 34B10, 34B15

URL: http://aaa.hindawi.com/volume-6/S1085337501000550.html 
At this point we introduce some notation. We will denote by $C[a, b]\left(C^{1}[a, b]\right)$ the classical space of the continuous (continuously differentiable) functions defined from $[a, b]$ into $\mathbb{R}$. The norm in $C[a, b]$ is denoted by $|\cdot|_{\infty}$. Also we will denote by $L^{1}(a, b)$ the space of measurable real-valued functions (equivalence classes) whose absolute value is Lebesgue integrable on $(a, b)$. The Brouwer and Leray-Schauder degree will be, respectively, denoted by $\operatorname{deg}_{\mathrm{B}}$ and $\operatorname{deg}_{\mathrm{LS}}$.

This paper is organized as follows. In Section 2, we provide an abstract formulation for problem (1.1) and we establish a general continuation theorem for the solvability of that problem in the same spirit of [6, 14]. Using this result, in Section 3 we obtain two existence theorems. Thus in Theorem 3.1 of Section 3 we generalize [3, Theorem 2.2] obtained for the linear operator within the framework of the coincidence degree of Mawhin [17]. Our second existence result in Section 3 is closer in spirit to the existence results of [6].

To illustrate those results we state next some consequences of them for the particular situation containing the one-dimensional $p$-Laplace operator, $\left(\phi_{p}\left(u^{\prime}\right)\right)^{\prime}$, where $\phi_{p}, p>1$, is the homeomorphism from $\mathbb{R}$ onto $\mathbb{R}$ defined by

$$
\phi_{p}(s)=|s|^{p-2} s \quad \text { for } s \neq 0, \phi_{p}(0)=0 .
$$

THEOREM 1.1. Consider the problem

$$
\begin{gathered}
\left(\phi_{p}\left(u^{\prime}\right)\right)^{\prime}=f\left(t, u, u^{\prime}\right), \quad t \in(a, b), \\
u^{\prime}(a)=0, \quad u(\eta)=u(b),
\end{gathered}
$$

where $\eta \in(a, b)$. Assume that $f:[a, b] \times \mathbb{R} \times \mathbb{R} \mapsto \mathbb{R}$ is continuous and satisfies the following conditions.

(i) There are nonnegative functions $d_{1}, d_{2}$, and $r$ in $L^{1}(a, b)$ such that

$$
|f(t, u, v)| \leq d_{1}(t)|u|^{p-1}+d_{2}(t)|v|^{p-1}+r(t),
$$

for a.e. $t \in[a, b]$ and all $u, v \in \mathbb{R}$.

(ii) There exists $u_{0}>0$ such that for all $|u|>u_{0}$, it holds that

$$
|f(t, u, v)| \geq \Lambda|u|^{p-1}-A|v|^{p-1}-B
$$

where $\Lambda>0$, and $A, B \geq 0$ are fixed constants.

(iii) There is $R>0$ such that for all $|u|>R$, either

$$
u f(t, u, 0)>0 \text { for a.e. } t \in[a, b],
$$

or

$$
u f(t, u, 0)<0 \text { for a.e. } t \in[a, b] .
$$


Then, if

$$
\left(\frac{A}{\Lambda}+(b-a)^{(p-1)}\right)\left\|d_{1}\right\|_{L^{1}(a, b)}+\left\|d_{2}\right\|_{L^{1}(a, b)}<1,
$$

it follows that problem (1.4) has at least one solution with $u \in C^{1}[a, b]$.

THEOREM 1.2. Let $f:[a, b] \times \mathbb{R}^{2} \rightarrow \mathbb{R}$ be a function satisfying Carathéodory's conditions. Assume that there exist functions $d_{1}, d_{2}, r$ in $L^{1}(a, b)$ such that

$$
|f(t, u, v)| \leq d_{1}(t)|u|^{p-1}+d_{2}(t)|v|^{p-1}+r(t)
$$

for a.e. $t \in[a, b]$ and all $(u, v) \in \mathbb{R}^{2}$. Suppose further that there exists an $M>0$ such that

$$
\begin{aligned}
& f(t, u, v)>0, \quad \text { if } u>M \forall t \in[a, b], v \in \mathbb{R}, \\
& f(t, u, v)<0, \quad \text { if } u<-M \forall t \in[a, b], v \in \mathbb{R} .
\end{aligned}
$$

Then the boundary value problem (1.4) has at least one solution in $C^{1}[a, b]$ provided that

$$
(b-a)^{(p-1)}\left\|d_{1}\right\|_{L^{1}(a, b)}+\left\|d_{2}\right\|_{L^{1}(a, b)}<1 .
$$

The proofs of Theorems 1.1 and 1.2 are direct applications of Theorems 3.1 and 3.2 , respectively.

In Section 4, we prove some existence results with the help of time-mapping techniques as in $[6,7,8]$. Our main purpose here is to obtain existence results with one-sided growth restrictions for the three-point boundary value problem. Conditions of this type have been considered by Schmitt [20], Mawhin and Ward [18], and Fernandes and Zanolin [4] for the periodic case and the second-order linear differential operator, by de Figueiredo and Ruf in [1] for the second-order linear differential operator and Neumann boundary conditions, and by Manásevich and Zanolin in [16] for the one-dimensional $p$-Laplacian and Dirichlet boundary value conditions.

We introduce here a technical condition for the homeomorphism $\phi$ which will be used in Section 4 in order to guarantee some properties of the time-mapping for non-homogeneous operators (see [6]).

We say that $\phi$ satisfies the lower $\sigma$-condition if for any $\sigma>1$,

$$
\liminf _{s \rightarrow+\infty} \frac{\phi(\sigma s)}{\phi(s)}>1
$$

We end this section by stating a theorem which is a consequence of Theorem 4.3 in Section 4 and which illustrates the type of results that we will obtain in that section. We first give the following definitions. For $q \in L^{1}(a, b)$, we set

$$
q^{m}:=\sup _{s \in(a, b]} \frac{1}{s-a} \int_{a}^{s} q(t) d t, \quad q_{m}:=\inf _{s \in(a, b]} \frac{1}{s-a} \int_{a}^{s} q(t) d t .
$$


Also from [2], we recall that the number $\pi_{p}$, which will be used below, is defined by

$$
\pi_{p}:=2(p-1)^{1 / p} \int_{0}^{1} \frac{d s}{\left(1-s^{p}\right)^{1 / p}}=2(p-1)^{1 / p} \frac{(\pi / p)}{\sin (\pi / p)} .
$$

THEOREM 1.3. Consider the problem

$$
\begin{gathered}
\left(\phi_{p}\left(u^{\prime}\right)\right)^{\prime}+g(u)=q(t), \\
u^{\prime}(a)=0, \quad u(\eta)=u(b),
\end{gathered}
$$

where $\eta \in(a, b)$ and $q \in L^{1}(a, b)$, with $q^{m}, q_{m}$ defined in (1.15) such that

$$
-\infty<q_{m}, \quad q^{m}<+\infty
$$

The function $g: \mathbb{R} \mapsto \mathbb{R}$ is continuous and satisfies

$$
\begin{aligned}
& g(s) \geq q^{m}>0 \quad \text { for } s \geq d>0, \\
& g(s) \leq q_{m}<0 \quad \text { for } s \leq-d .
\end{aligned}
$$

Suppose also that $G(s):=\int_{0}^{s} g(t) d t$ satisfies

$$
\liminf _{s \rightarrow+\infty} \frac{p G(s)}{|s|^{p}} \leq k<\left(\frac{\pi_{p}}{b-a}\right)^{p},
$$

then problem (1.17) has at least one solution.

The proof of this theorem will be given in Section 4 .

\section{Abstract formulation and a deformation lemma}

We begin this section by developing a general continuation theorem for the solvability of problem (1.1). Assume that $f^{*}:[a, b] \times \mathbb{R} \times \mathbb{R} \times[0,1] \mapsto \mathbb{R}$ satisfies the Carathéodory conditions, that is, $f^{*}(\cdot, s, r, \lambda)$ is measurable for all $(s, r, \lambda) \in \mathbb{R} \times \mathbb{R} \times[0,1], f^{*}(t, \cdot, \cdot, \cdot)$ is continuous for a.e. $t \in[a, b]$, and for each $R>0$ there exists a Lebesgue integrable function $\mu_{R}:[a, b] \mapsto \mathbb{R}$ such that $\left|f^{*}(t, s, r, \lambda)\right| \leq \mu_{R}(t)$ for a.e. $t \in[a, b]$ and all $(s, r, \lambda)$ with $|s| \leq R$, $|r| \leq R$, and $\lambda \in[0,1]$. Furthermore, suppose that $f^{*}(t, s, r, 1)=f(t, s, r)$ for all $(t, s, r) \in[a, b] \times \mathbb{R} \times \mathbb{R}$.

For $\lambda \in(0,1]$, consider the problem

$$
\begin{aligned}
& \left(\phi\left(\frac{u^{\prime}}{\lambda}\right)\right)^{\prime}=f^{*}\left(t, u, u^{\prime}, \lambda\right), \\
& u^{\prime}(a)=0, \quad u(\eta)=u(b),
\end{aligned}
$$

and let $\Omega \subset C^{1}[a, b]$ be an open bounded set. We have the following continuation lemma. 
LeMma 2.1. Assume that

(i) there is no solution $u$ to (2.1), $0<\lambda<1$, such that $u \in \partial \Omega$,

(ii) the equation

$$
F(s):=\int_{\eta}^{b} \phi^{-1}\left(\int_{a}^{\tau} f^{*}(t, s, 0,0) d t\right) d \tau=0
$$

has no solution on $\partial \Omega \cap \mathbb{R}$,

(iii) the Brouwer degree

$$
\operatorname{deg}_{\mathrm{B}}[F, \Omega \cap \mathbb{R}, 0] \neq 0 .
$$

Then, problem (1.1) has a solution in $\bar{\Omega}$.

Proof. If (1.1) has a solution in $\partial \Omega$, then there is nothing to prove, hence we suppose that (1.1) has no solutions belonging to $\partial \Omega$. This assumption combined with (i) implies that there are no solutions to (2.1) in $\partial \Omega$ for $0<\lambda \leq 1$.

We show next that $(2.1)$, for $\lambda \in(0,1]$, is equivalent to an abstract equation. Indeed, define the operator $\Psi^{*}: C^{1}[a, b] \times[0,1] \mapsto C^{1}[a, b]$, by

$$
\begin{aligned}
\Psi^{*}(u, \lambda)(t):= & u(a)+\int_{\eta}^{b} \phi^{-1}\left(\int_{a}^{s} f^{*}\left(t, u(t), u^{\prime}(t), \lambda\right) d t\right) d s \\
& +\lambda \int_{a}^{t} \phi^{-1}\left(\int_{a}^{s} f^{*}\left(\tau, u(\tau), u^{\prime}(\tau), \lambda\right) d \tau\right) d s .
\end{aligned}
$$

We note that for $u \in C^{1}[a, b]$ and $\lambda \in[0,1]$, it holds that $f^{*}\left(\cdot, u(\cdot), u^{\prime}(\cdot), \lambda\right) \in$ $L^{1}$. Thus the mapping $s \mapsto \int_{a}^{s} f^{*}\left(\tau, u(\tau), u^{\prime}(\cdot), \lambda\right) d \tau$ is absolutely continuous and hence the operator $\Psi^{*}$ is well defined since $\phi^{-1}\left(\int_{a}^{s} f^{*}\left(\tau, u(\tau), u^{\prime}(\tau), \lambda\right) d \tau\right)$ is continuous.

Now, by integrating the equation in (2.1) and using the boundary conditions, we find that if $u$ is a solution of (2.1), then it satisfies

$$
u=\Psi^{*}(u, \lambda),
$$

together with

$$
\int_{\eta}^{b} \phi^{-1}\left(\int_{a}^{s} f^{*}\left(\tau, u(\tau), u^{\prime}(\tau), \lambda\right) d \tau\right) d s=0 .
$$

Next, for $\lambda \in(0,1]$, assume that $u$ is a solution to $(2.5)$, that is, $u$ satisfies

$$
\begin{aligned}
u(t)= & u(a)+\int_{\eta}^{b} \phi^{-1}\left(\int_{a}^{s} f^{*}\left(t, u(t), u^{\prime}(t), \lambda\right) d t\right) d s \\
& +\lambda \int_{a}^{t} \phi^{-1}\left(\int_{a}^{s} f^{*}\left(\tau, u(\tau), u^{\prime}(\tau), \lambda\right) d \tau\right) d s
\end{aligned}
$$


for all $t \in[a, b]$. Differentiating (2.7), we find that

$$
\phi\left(\frac{u^{\prime}(t)}{\lambda}\right)=\int_{a}^{t} f^{*}\left(\tau, u(\tau), u^{\prime}(\tau), \lambda\right) d \tau,
$$

and hence $\phi\left(u^{\prime}(t) / \lambda\right)$ is absolutely continuous. By differentiating (2.8) then $u$ satisfies the first equation in (2.1). Also from (2.8), $u^{\prime}(a)=0$, and evaluating (2.7) for $t=a$, we find that

$$
\int_{\eta}^{b} \phi^{-1}\left(\int_{a}^{s} f^{*}\left(t, u(t), u^{\prime}(t), \lambda\right) d t\right) d s=0 .
$$

Hence, from (2.7)

$$
u(b)-u(\eta)=\lambda \int_{\eta}^{b} \phi^{-1}\left(\int_{a}^{s} f^{*}\left(t, u(t), u^{\prime}(t), \lambda\right) d t\right) d s=0 .
$$

This shows that, for $\lambda \in(0,1]$, any solution of (2.5) (equivalently (2.7)) is actually a solution to the boundary value problem (2.1).

Setting $\Psi(u):=\Psi^{*}(u, 1)$, we observe that $u$ is a solution of (1.1) if and only if it is a fixed point of $\Psi$.

Standard arguments show that $\Psi^{*}$ is a completely continuous operator. Moreover, assumption (i) of Lemma 2.1 can be restated as

$$
u \neq \Psi^{*}(u, \lambda) \quad \forall u \in \partial \Omega, \forall \lambda \in(0,1] .
$$

We show next that this is also true for $\lambda=0$. We note from $(2.4)$ that $\Psi^{*}(u, 0)(t)$, $t \in[a, b]$, is a real constant for each $u \in C^{1}[a, b]$. Thus, if for some $u \in \partial \Omega$,

$$
u=\Psi^{*}(u, 0),
$$

then, for all $t \in[a, b]$, we have that $u(t)=s \in \mathbb{R}$, and so $u(a)=s$. Hence, from (2.7), with $\lambda=0$,

$$
s=s+\int_{\eta}^{b} \phi^{-1}\left(\int_{a}^{\tau} f(t, s, 0,0) d t\right) d \tau=s+F(s),
$$

which implies that $F(s)=0$, for $s \in \mathbb{R} \cap \partial \Omega$, contradicting assumption (ii) of Lemma 2.1. In this manner we have verified that

$$
u \neq \Psi^{*}(u, \lambda) \quad \forall u \in \partial \Omega, \forall \lambda \in[0,1] .
$$

Then, from the homotopy invariance property of the Leray-Schauder degree, it follows that

$$
\begin{aligned}
\operatorname{deg}_{\mathrm{LS}} & \left(I-\Psi^{*}(\cdot, 1), \Omega, 0\right) \\
= & \operatorname{deg}_{\mathrm{LS}}\left(I-\Psi^{*}(\cdot, 0), \Omega, 0\right)=\operatorname{deg}_{\mathrm{B}}\left(I-\left.\Psi^{*}(\cdot, 0)\right|_{\mathbb{R}}, \Omega_{0}, 0\right) \\
= & \operatorname{deg}_{\mathrm{B}}\left(F, \Omega_{0}, 0\right) \neq 0,
\end{aligned}
$$

where $\Omega_{0}=\Omega \cap \mathbb{R}$. In this form we obtain that the mapping $\Psi=\Psi^{*}(\cdot, 1)$ has at least one fixed point in $\Omega$ and hence that problem (1.1) has at least one solution in $\Omega$. 


\section{First existence results}

Consider the boundary value problem (1.1) given in Section 1 . We have the following result.

Theorem 3.1. Assume that $f:[a, b] \times \mathbb{R} \times \mathbb{R} \mapsto \mathbb{R}$ in problem (1.1) is continuous and satisfies the following conditions.

(i) There are nonnegative functions $d_{1}, d_{2}$, and $r$ in $L^{1}(a, b)$ such that

$$
|f(t, u, v)| \leq d_{1}(t) \phi(|u|)+d_{2}(t) \phi(|v|)+r(t),
$$

for a.e. $t \in[a, b]$ and all $u, v \in \mathbb{R}$.

(ii) There exists $u_{0}>0$ such that for all $|u|>u_{0}$, for all $t \in[a, b]$ and $v \in \mathbb{R}$, it holds that

$$
|f(t, u, v)| \geq \Lambda \phi(|u|)-A \phi(|v|)-B
$$

where $\Lambda>0$, and $A, B \geq 0$.

(iii) There is $R>0$ such that for all $|u|>R$, either

$$
u f(t, u, 0)>0 \quad \forall t \in[a, b]
$$

or

$$
u f(t, u, 0)<0 \quad \forall t \in[a, b] .
$$

If, in addition $\left\|d_{2}\right\|_{L^{1}(a, b)}<1$, and the function $\Theta:[0,+\infty) \mapsto[0,+\infty)$, defined by

$$
\begin{aligned}
\Theta(z):= & \phi^{-1}\left(\frac{A\left\|d_{1}\right\|_{L^{1}(a, b)}}{\Lambda\left(1-\left\|d_{2}\right\|_{L^{1}(a, b)}\right)} \phi(z)+\frac{A\|r\|_{L^{1}(a, b)}}{\Lambda\left(1-\left\|d_{2}\right\|_{L^{1}(a, b)}\right)}+\frac{B}{\Lambda}\right) \\
& +(b-a) \phi^{-1}\left(\frac{\left\|d_{1}\right\|_{L^{1}(a, b)}}{\left(1-\left\|d_{2}\right\|_{L^{1}(a, b)}\right)} \phi(z)+\frac{\|r\|_{L^{1}(a, b)}}{\left(1-\left\|d_{2}\right\|_{L^{1}(a, b)}\right)}\right)
\end{aligned}
$$

satisfies

$$
\limsup _{z \rightarrow+\infty} \frac{\Theta(z)}{z}<1
$$

then, problem (1.1) has at least one solution $u \in C^{1}[a, b]$.

Proof. We consider problem (2.1) with $f^{*}\left(t, u, u^{\prime}, \lambda\right)=f\left(t, u, u^{\prime}\right)$ for all $\lambda \in$ $[0,1]$, that is, we consider the problem

$$
\begin{gathered}
\left(\phi\left(\frac{u^{\prime}}{\lambda}\right)\right)^{\prime}=f\left(t, u, u^{\prime}\right), \\
u^{\prime}(a)=0, \quad u(\eta)=u(b),
\end{gathered}
$$


and show that Lemma 2.1(i), (ii), and (iii) are satisfied. Let $u$ be a solution to (3.7), for $\lambda \in(0,1)$. Then, by (3.1),

$$
\left|\left(\phi\left(\frac{u^{\prime}(t)}{\lambda}\right)\right)^{\prime}\right| \leq d_{1}(t) \phi(|u(t)|)+d_{2}(t) \phi\left(\left|u^{\prime}(t)\right|\right)+r(t),
$$

and hence, since $\lambda \in(0,1)$, and by integration, we find that

$$
\begin{aligned}
\phi\left(\left|u^{\prime}(t)\right|\right) & \leq \phi\left(\frac{\left|u^{\prime}(t)\right|}{\lambda}\right) \\
& \leq \phi\left(|u|_{\infty}\right)\left\|d_{1}\right\|_{L^{1}(a, b)}+\phi\left(\left|u^{\prime}\right|_{\infty}\right)\left\|d_{2}\right\|_{L^{1}(a, b)}+\|r\|_{L^{1}(a, b)} .
\end{aligned}
$$

Therefore

$$
\phi\left(\left|u^{\prime}\right|_{\infty}\right) \leq \frac{\left\|d_{1}\right\|_{L^{1}(a, b)}}{1-\left\|d_{2}\right\|_{L^{1}(a, b)}} \phi\left(|u|_{\infty}\right)+\frac{\|r\|_{L^{1}(a, b)}}{1-\left\|d_{2}\right\|_{L^{1}(a, b)}},
$$

which yields

$$
\left|u^{\prime}\right|_{\infty} \leq \phi^{-1}\left(\frac{\left\|d_{1}\right\|_{L^{1}(a, b)}}{1-\left\|d_{2}\right\|_{L^{1}(a, b)}} \phi\left(|u|_{\infty}\right)+\frac{\|r\|_{L^{1}(a, b)}}{1-\left\|d_{2}\right\|_{L^{1}(a, b)}}\right) .
$$

In our next argument assume first that there is $\tau_{0} \in[a, b]$, such that $\left|u\left(\tau_{0}\right)\right| \leq$ $u_{0}$. Then, since for any $t \in(a, b)$

$$
|u(t)|=\left|u\left(\tau_{0}\right)+\int_{\tau_{0}}^{t} u^{\prime}(s) d s\right| \leq\left|u\left(\tau_{0}\right)\right|+\left|u^{\prime}\right|_{\infty}(b-a),
$$

by (3.11), we find that

$$
\begin{aligned}
|u|_{\infty} & \leq u_{0}+(b-a) \phi^{-1}\left(\frac{\left\|d_{1}\right\|_{L^{1}(a, b)}}{1-\left\|d_{2}\right\|_{L^{1}(a, b)}} \phi\left(|u|_{\infty}\right)+\frac{\|r\|_{L^{1}(a, b)}}{1-\left\|d_{2}\right\|_{L^{1}(a, b)}}\right) \\
& \leq u_{0}+\Theta\left(|u|_{\infty}\right) .
\end{aligned}
$$

Suppose next that $|u(t)|>u_{0}$ for all $t \in[a, b]$. Then since $u(\eta)=u(b)$, there must be $\tau_{1} \in(\eta, b)$ such that $u^{\prime}\left(\tau_{1}\right)=0$, that is,

$$
\int_{a}^{\tau_{1}} f\left(t, u(t), u^{\prime}(t)\right) d t=0
$$

which in turn implies that there is $\tau_{0} \in\left(a, \tau_{1}\right)$, such that $f\left(\tau_{0}, u\left(\tau_{0}\right), u^{\prime}\left(\tau_{0}\right)\right)=0$. Hence from (3.2), we obtain

$$
\phi\left(\left|u\left(\tau_{0}\right)\right|\right) \leq \tilde{A} \phi\left(\left|u^{\prime}\left(\tau_{0}\right)\right|\right)+\tilde{B} \leq \tilde{A} \phi\left(\left|u^{\prime}\right|_{\infty}\right)+\tilde{B},
$$


where $\tilde{A}=A / \Lambda, \tilde{B}=B / \Lambda$. This implies

$$
\left|u\left(\tau_{0}\right)\right| \leq \phi^{-1}\left(\tilde{A} \phi\left(\left|u^{\prime}\right|_{\infty}\right)+\tilde{B}\right) .
$$

Now from (3.12) and (3.16), we find that

$$
|u|_{\infty} \leq \phi^{-1}\left(\tilde{A} \phi\left(\left|u^{\prime}\right|_{\infty}\right)+\tilde{B}\right)+(b-a)\left|u^{\prime}\right|_{\infty},
$$

and thus by (3.10) and (3.11), it follows that

$$
|u|_{\infty} \leq \Theta\left(|u|_{\infty}\right)
$$

Therefore by (3.6), in both situations we obtain the existence of $z_{0}>0$ such that $|u|_{\infty} \leq z_{0}$, and thus by (3.11) there is an $R_{0}>R$ ( $R$ defined by hypothesis (iii)) so that condition (i) of Lemma 2.1 is satisfied for $\Omega=B(0, \tilde{R}) \subset C^{1}([a, b])$, for all $\tilde{R} \geq R_{0}$. Since hypothesis (iii) implies that conditions (ii) and (iii) of Lemma 2.1 are satisfied for $\tilde{R}$ large, the proof of the theorem is completed.

In our second application we consider the boundary value problem

$$
\begin{aligned}
& \left(\phi\left(u^{\prime}\right)\right)^{\prime}+f\left(t, u, u^{\prime}\right)=q(t), \\
& u^{\prime}(a)=0, \quad u(\eta)=u(b),
\end{aligned}
$$

where $\eta \in(a, b)$.

Theorem 3.2. Assume that $q \in L^{1}(a, b)$, and that $q^{m}$ and $q_{m}$ defined in (1.15) satisfy (1.18). Suppose also that $f:[a, b] \times \mathbb{R} \times \mathbb{R} \mapsto \mathbb{R}$ is Carathéodory and satisfies the following conditions.

(i) There are nonnegative functions $d_{1}, d_{2}$, and $r$ in $L^{1}(a, b)$ such that

$$
|f(t, u, v)| \leq d_{1}(t) \phi(|u|)+d_{2}(t) \phi(|v|)+r(t),
$$

for a.e. $t \in[a, b]$ and all $u, v \in \mathbb{R}$.

(ii) There exists $d>0$ such that

$$
\begin{array}{ll}
f(t, u, v)>q^{m} & \text { for } u \geq d, \\
f(t, u, v)<q_{m} & \text { for } u \leq-d
\end{array}
$$

holds for a.e. $t \in[a, b]$ and all $v \in \mathbb{R}$.

(iii) There is $R>0$ such that for all $|u|>R$, either

$$
u f(t, u, 0)>0 \text { for a.e. } t \in[a, b] \text {, }
$$

or

$$
u f(t, u, 0)<0 \text { for a.e. } t \in[a, b] .
$$


Furthermore, let

$$
\Gamma(z):=(b-a) \phi^{-1}\left(\frac{\left\|d_{1}\right\|_{L^{1}(a, b)}}{\left(1-\left\|d_{2}\right\|_{L^{1}(a, b)}\right)} \phi(z)+\frac{\|\tilde{r}\|_{L^{1}(a, b)}}{\left(1-\left\|d_{2}\right\|_{L^{1}(a, b)}\right)}\right),
$$

where $\tilde{r}(t)=r(t)+|q(t)|$. Then if

$$
\limsup _{z \rightarrow+\infty} \frac{\Gamma(z)}{z}<1
$$

it follows that problem (3.19) has at least one solution $u \in C^{1}[a, b]$.

Remark 3.3. We note that if $q^{m}>0$ and $q_{m}<0$ in Theorem 3.2, then the conditions in (ii) imply (3.22).

Proof. The proof is based again in Lemma 2.1 and thus we will show that conditions (i), (ii), and (iii) of that lemma are satisfied. Thus consider problem (2.1), where this time we take $f^{*}\left(t, u, u^{\prime}, \lambda\right):=q(t)-f\left(t, u, u^{\prime}\right)$, and thus we consider the problem

$$
\begin{gathered}
\left(\phi\left(\frac{u^{\prime}}{\lambda}\right)\right)^{\prime}=q(t)-f\left(t, u, u^{\prime}\right), \\
u^{\prime}(a)=0, \quad u(\eta)=u(b),
\end{gathered}
$$

where $\eta \in(a, b)$, and $\lambda \in(0,1)$. By (3.20), we obtain that

$$
|q(t)-f(t, u, v)| \leq d_{1}(t) \phi(|u|)+d_{2}(t) \phi(|v|)+\tilde{r}(t),
$$

and hence as in Theorem 3.1, from (3.26), we find that

$$
\left|u^{\prime}\right|_{\infty} \leq \phi^{-1}\left(\frac{\left\|d_{1}\right\|_{L^{1}(a, b)}}{1-\left\|d_{2}\right\|_{L^{1}(a, b)}} \phi\left(|u|_{\infty}\right)+\frac{\|\tilde{r}\|_{L^{1}(a, b)}}{1-\left\|d_{2}\right\|_{L^{1}(a, b)}}\right) .
$$

Next, let $u$ be a solution of (3.26) for some $\lambda \in(0,1)$. We claim that there is a $\tilde{t} \in[a, b]$, such that

$$
-d \leq u(\tilde{t}) \leq d
$$

Indeed, integrating the equation of (3.26), we find that

$$
\phi\left(\frac{u^{\prime}}{\lambda}\right)=\left(\int_{a}^{t} q(\tau) d \tau-\int_{a}^{t} f\left(\tau, u(\tau), u^{\prime}(t)\right) d \tau\right) .
$$

Hence if $u(t) \geq d$ for all $t \in[a, b]$, then from the first condition in hypothesis (ii), we find that

$$
\phi\left(\frac{u^{\prime}}{\lambda}\right)<\int_{a}^{t} q(\tau) d \tau-q^{m}(t-a) \leq 0, \quad \forall t \in[a, b],
$$

which cannot be because of the boundary condition $u(\eta)=u(b)$. 
Similarly, if $u(t) \leq-d$ for all $t \in[a, b]$, then from the second condition in (ii),

$$
\int_{a}^{t} f\left(t, u(t), u^{\prime}(t)\right) d t<q_{m}(t-a)
$$

and hence

$$
\phi\left(\frac{u^{\prime}}{\lambda}\right)>\int_{a}^{t} q(\tau) d \tau-q_{m}(t-a) \geq 0, \quad \forall t \in[a, b],
$$

which again cannot be. Hence in the case that the solution is a constant, say $u(t)=c$, then necessarily $|c| \leq d$.

In this form we find that

$$
|u|_{\infty} \leq d+\left|u^{\prime}\right|_{\infty}(b-a) .
$$

Combining this inequality with (3.28), it follows that

$$
|u|_{\infty} \leq d+\Gamma\left(|u|_{\infty}\right)
$$

and thus from (3.25) there must be a $z_{0}>0$ such that $|u|_{\infty} \leq z_{0}$. Hence combining with (3.28), we find that there is $R_{0}>R$ ( $R$ as in hypothesis (iii)), so that for all $\tilde{R} \geq R_{0}$ if $\Omega=B(0, \tilde{R}) \subset C^{1}([a, b])$, then for all $\lambda \in(0,1)$, problem (3.26) has no solution in $\partial \Omega$. Hence hypothesis (i) of Lemma 2.1 is satisfied. Also, by hypothesis (ii), it follows that

$$
\int_{a}^{t}(q(\tau)-f(\tau, \tilde{R}, 0)) d \tau<\int_{a}^{t}\left(q(\tau)-q^{m}\right) d \tau \leq 0, \quad \forall t \in(a, b],
$$

and thus $F(s)$ as defined in (2.2) satisfies $F(\tilde{R})<0$, for $\tilde{R}$ large enough. Similarly $F(-\tilde{R})>0$, and hence Lemma 2.1(ii) is satisfied.

Finally, condition (iii) of this theorem implies that Lemma 2.1(iii) is also satisfied with

$$
\operatorname{deg}_{\mathrm{B}}(F, B(0, \tilde{R}) \cap \mathbb{R}, 0)= \pm 1,
$$

for large $\tilde{R}$. This ends the proof of the theorem.

Remark 3.4. We note at this point the important fact that for the two theorems we have proved in this section there is no need of additional hypotheses on the function $\phi$ besides being an odd increasing homeomorphism.

\section{Existence results via time-mapping}

In this section we will consider the problem

$$
\begin{gathered}
\left(\phi\left(u^{\prime}\right)\right)^{\prime}+g(t, u)=q(t), \\
u^{\prime}(a)=0, \quad u(\eta)=u(b),
\end{gathered}
$$


where $\eta \in(a, b), g$ is Carathéodory, and $q \in L^{1}(a, b)$. In this respect the following obvious modification of Lemma 2.1 will be used.

Let $g^{*}:[a, b] \times \mathbb{R} \times[0,1] \mapsto \mathbb{R}$ be a function which satisfies the Carathéodory conditions and is such that

$$
g^{*}(t, s, 1)=g(t, s) \quad \forall(t, s) \in[a, b] \times \mathbb{R},
$$

and for $\lambda \in(0,1]$, consider the problem

$$
\begin{gathered}
\left(\phi\left(\frac{u^{\prime}}{\lambda}\right)\right)^{\prime}+g^{*}(t, u, \lambda)=q(t), \\
u^{\prime}(a)=0, \quad u(\eta)=u(b) .
\end{gathered}
$$

Lemma 4.1. Let $\Omega \subset C^{0}[a, b]$ be an open bounded set, such that

(i) there is no solution $u$ to (4.3), $0<\lambda<1$, such that $u \in \partial \Omega$,

(ii) the equation

$$
G(s):=\int_{\eta}^{b} \phi^{-1}\left(\int_{a}^{\tau}\left(q(t)-g^{*}(t, s, 0)\right) d t\right) d \tau=0
$$

has no solution on $\partial \Omega \cap \mathbb{R}$,

(iii) the Brouwer degree

$$
\operatorname{deg}_{\mathrm{B}}[G, \Omega \cap \mathbb{R}, 0] \neq 0 .
$$

Then, problem (4.3) has a solution in $\bar{\Omega}$.

In our following step we show that under certain conditions on $g^{*}$ solutions to (4.3) which are bounded from above or from below are in fact bounded. See $[18,19]$, for analogous results in the periodic case for the linear operator, that is, $\phi(s)=s$, and [6] for the Neumann case.

LeMma 4.2. Let $q^{m}$ and $q_{m}$ be defined as in (1.15) and suppose they satisfy (1.18). Assume that there exists $d>0$ such that

$$
\begin{array}{ll}
g^{*}(t, s, \lambda)>q^{m} & \text { for } s \geq d, \\
g^{*}(t, s, \lambda)<q_{m} & \text { for } s \leq-d
\end{array}
$$

holds for a.e. $t \in[a, b]$ and all $\lambda \in[0,1]$. Then,

(i) for any solution $u$ to (4.3), for $\lambda \in(0,1)$, there exists $a \tilde{t} \in[a, b]$ such that

$$
-d \leq u(\tilde{t}) \leq d
$$

(ii) for any solution $u$ to (4.3), for $\lambda \in(0,1)$, we have that for each $R \geq d$ there is a $\rho(R) \geq R$, such that

$$
\max u \leq R \quad \text { or } \quad \min u \geq-R \quad \text { implies }|u|_{\infty} \leq \rho(R),
$$


(iii) if there exists $R \geq d$ such that there is no solution $u$ to (4.3), with $\lambda \in(0,1)$ such that $\max u=R,(\min u=-R)$, then problem (4.1) has at least one solution $\tilde{u}$ with $\max \tilde{u} \leq R(\min \tilde{u} \geq-R)$.

Proof. Let $u$ be a solution to (4.3) for some $\lambda \in(0,1)$. Since the proof of (i) is entirely similar to that of (3.29) of Theorem 3.2, it will be omitted. Also, and as in that theorem, we continue the proof assuming that $u$ is a nonconstant solution.

To prove (ii) we only consider the case $\max u \leq R$, since the argument in the case $\min u \geq-R$ is completely similar. Thus suppose $t_{1}$ and $t_{2}$ are, respectively, two points in $[a, b]$ where $u$ reaches its absolute maximum and minimum. We note that $t_{1}$ and $t_{2}$ belong to $[a, b)$ and thus $u^{\prime}\left(t_{1}\right)=0$, and $u^{\prime}\left(t_{2}\right)=0$. We assume $t_{1}<t_{2}$, with a similar argument for the other case. Integrating the equation of (4.3) on $\left[t_{1}, t_{2}\right]$, we find that

$$
\int_{t_{1}}^{t_{2}} g^{*}(\tau, u(\tau), \lambda) d \tau=\int_{t_{1}}^{t_{2}} q(\tau) d \tau
$$

Let $A=\left\{t \in\left[t_{1}, t_{2}\right]: u(t)<-d\right\}$ and $B=\left\{t \in\left[t_{1}, t_{2}\right]:-d \leq u(t) \leq R\right\}$. Then, by (4.9) and hypothesis (4.6),

$$
\begin{aligned}
\int_{A} \mid g^{*} & (\tau, u(\tau), \lambda)-q_{m} \mid d \tau \\
& =\int_{A}\left(q_{m}-g^{*}(\tau, u(\tau), \lambda)\right) d \tau \\
& =\int_{A} q_{m} d \tau-\int_{t_{1}}^{t_{2}} q(\tau) d \tau+\int_{B}\left(g^{*}(\tau, u(\tau), \lambda)-q_{m}\right) d \tau+\int_{B} q_{m} d \tau \\
& =q_{m}\left(t_{2}-t_{1}\right)-\int_{t_{1}}^{t_{2}} q(\tau) d \tau+\int_{B}\left(g^{*}(\tau, u(\tau), \lambda)-q_{m}\right) d \tau
\end{aligned}
$$

which in turn implies that

$$
\begin{aligned}
& \int_{t_{1}}^{t_{2}}\left|g^{*}(\tau, u(\tau), \lambda)-q_{m}\right| d \tau \\
& \quad \leq\left(\left|q_{m}\right|+\left|q^{m}\right|\right)(b-a)+2 \int_{B}\left|g^{*}(\tau, u(\tau), \lambda)-q_{m}\right| d \tau .
\end{aligned}
$$

Since $g^{*}$ satisfies the Carathéodory conditions, we find that

$$
\left|g^{*}(t, s, \lambda)\right| \leq \mu(t), \quad \text { a.e. } t \in[a, b], s \in[-d, R] \text { and all } \lambda \in[0,1],
$$

and where $\mu=\mu_{R} \in L^{1}(a, b)$. Then, the last integral in (4.11) can be bounded from above by $\int_{B}\left(\mu(t)-q_{m}\right) d t$, and thus

$$
\int_{t_{1}}^{t_{2}}\left|g^{*}(\tau, u(\tau), \lambda)-q_{m}\right| d \tau \leq\left(2\left|q_{m}\right|+q^{m}\right)(b-a)+|\mu|_{1}:=C_{1}(R) .
$$


Now, by integrating the equation of (4.3) on $\left[t_{1}, t\right]$, we find first that

$$
\left|\phi\left(\frac{u^{\prime}}{\lambda}\right)\right| \leq\left|\int_{t_{1}}^{t} q(\tau) d \tau\right|+\int_{t_{1}}^{t}\left|g^{*}(\tau, u(\tau), \lambda)\right| d \tau,
$$

and then, using (4.13), we find a constant $C_{2}(R)$ such that

$$
\left|u^{\prime}(t)\right| \leq C_{2}(R) \quad \forall t \in\left[t_{1}, t_{2}\right]
$$

We observe that $|u|_{\infty}$ is reached at $t_{1}$ or $t_{2}$. Also we note that there must be a point $t_{3} \in\left(t_{1}, t_{2}\right)$ such that $\left|u\left(t_{3}\right)\right| \leq d$. Thus by integrating (4.15) from $t_{1}$ to $t_{3}$, and assuming first that $|u|_{\infty}$ is reached at $t_{1}$, we obtain that

$$
|u|_{\infty}=\left|u\left(t_{1}\right)\right| \leq d+C_{2}(R)(b-a):=\rho(R),
$$

where, without loss of generality, we have taken $\rho(R) \geq R$. Since a similar argument applies if $|u|_{\infty}$ is reached at $t_{2}$, the proof of (ii) is completed.

Finally we prove (iii) by using Lemma 4.1 . Let $R \geq d$ be such that there is no solution $u$ to $(4.3)$, with $\lambda \in(0,1)$ and $\max u=R$ (the other case being analogous). Let $\rho(R)$ be the bound given in (ii), and define

$$
R_{1}:=\eta(R)+1
$$

Define also $\Omega \subset C^{0}[a, b]$ by

$$
\Omega=\left\{u \in C^{0}[a, b]:-R_{1}<u(t)<R\right\} .
$$

For $\lambda \in(0,1)$, suppose $u$ is a solution to (4.3). We claim that $u \notin \partial \Omega$. Indeed, if $u \in \bar{\Omega}$, then $-R_{1} \leq u(t) \leq R$ for all $t \in[a, b]$ and thus by our hypotheses, $u(t)<R$. Now from the choice of $R_{1}>\rho(R) \geq R$ we have that $u(t)>-R_{1}$, concluding that $-R_{1}<u(t)<R$ for all $t \in[a, b]$. Thus $u \in \Omega$ and (i) of Lemma 4.1 is satisfied.

Next we note that $\Omega \cap \mathbb{R}=\left(-R_{1}, R\right)$ and $\partial \Omega \cap \mathbb{R}=\left\{-R_{1}, R\right\}$. Also by hypothesis (4.6), it follows that

$$
\int_{a}^{t}\left(q(\tau)-g^{*}(\tau, R, 0)\right) d \tau<\int_{a}^{t} q(\tau) d \tau-q^{m} \leq 0 \quad \forall t \in[a, b],
$$

and thus $G(R)<0$. Similarly $G(-R)>0$. Hence, Lemma 4.1(ii) holds and also (iii) of that lemma is satisfied with

$$
\operatorname{deg}_{\mathrm{B}}(G, \Omega \cap \mathbb{R}, 0)=-1
$$

We conclude from Lemma 4.1 that there is at least one solution $u$ to $(4.1) \in \bar{\Omega}$ with $\max u \leq R$. 
We continue by reviewing some basic facts concerning time-mappings. Thus consider the equation

$$
\left(\phi\left(u^{\prime}\right)\right)^{\prime}+h(u)=0
$$

where $h: \mathbb{R} \mapsto \mathbb{R}$ satisfies $\lim _{s \rightarrow+\infty} h(s) \operatorname{sgn}(s)=+\infty$. This equation can be equivalently written as the autonomous system

$$
u^{\prime}=\phi^{-1}(y), \quad y^{\prime}=-h(u) .
$$

Set

$$
H(s)=\int_{0}^{s} h(t) d t, \quad \Phi^{*}(s)=\int_{0}^{s} \phi^{-1}(t) d t,
$$

and suppose that $(u(t), y(t))$ is a solution to (4.22) with $(u(0), y(0))=(0, S)$, $S>0$. Then, it holds that

$$
H(u(t))+\Phi^{*}(y(t))=\Phi^{*}(S)
$$

for all $t \in \mathbb{R}$. Let $d_{0}>0$ be such that

$$
h(s) s>0 \quad \forall|s| \geq d_{0}
$$

and take $d_{1} \geq d_{0}$ such that

$$
\max \left\{H(s):|s| \leq d_{0}\right\}<\min \left\{H\left(-d_{1}\right), H\left(d_{1}\right)\right\} .
$$

Now, if the constant $S$ selected above satisfies

$$
\Phi^{*}(S) \geq \max \left\{H\left(-d_{1}\right), H\left(d_{1}\right)\right\},
$$

then the corresponding solution $z$ is unique, defined in $\mathbb{R}$ and periodic. Let $T>0$ be the first maximum point of $u$ on $(0,+\infty)$. Then $u_{\max }=u(T)=R \geq d_{1}$ so that

$$
H(u(t))+\Phi^{*}(y(t))=H(R)
$$

for all $t \in \mathbb{R}$. Hence $y(t)>0$ and from (4.22), $u^{\prime}(t)>0$ for all $t \in(0, T)$. Thus $0<u(t)<R$ for all $t \in(0, T)$, with $y(T)=0$. Therefore we obtain

$$
y(t)=\Psi_{\mathrm{r}}(H(R)-H(u(t))),
$$

where $\Psi_{\mathrm{r}}$ denotes the right inverse of $\Phi^{*}$, that is, the inverse of the restriction of $\Phi^{*}$ to $[0,+\infty)$. Then, from the first equation in system (4.22),

$$
u^{\prime}(t)=\phi^{-1}\left(\Psi_{\mathrm{r}}(H(R)-H(u(t)))\right),
$$

so that

$$
\frac{u^{\prime}(t)}{\phi^{-1}\left(\Psi_{\mathrm{r}}(H(R)-H(u(t)))\right)}=1
$$


206 Solvability for nonlinear three point

for all $t \in[a, b)$. Integrating (4.31) on $[0, T)$ we obtain

$$
T_{h}(R):=T=\int_{0}^{R} \frac{d u}{\phi^{-1}\left(\Psi_{\mathrm{r}}(H(R)-H(u))\right)} .
$$

We call this function $T_{h}$ so far defined for large positive values of $R$, the timemapping of $h$ with respect to $\phi$, or simply the time-mapping of $h$. In a similar form we can define $T_{h}$ for large negative values. Indeed by assuming that $u_{\min }=$ $u(-\tilde{T})=-\tilde{R}, \tilde{R}>0$, we have

$$
\tilde{T}=T_{h}(-\tilde{R}):=\int_{-\tilde{R}}^{0} \frac{d u}{\left|\phi^{-1}\left(\Psi_{1}(H(-\tilde{R})-H(u))\right)\right|},
$$

where now $\Psi_{1}$ denotes the left inverse of $\Phi^{*}$. We note that in our case $\Psi_{1}(s)=$ $-\Psi_{\mathrm{r}}(s)$, since we are assuming $\phi$ is odd.

THEOREM 4.3. Assume that the odd increasing homeomorphism $\phi$ from $\mathbb{R}$ onto $\mathbb{R}$ satisfies the lower $\sigma$-condition. Let $q \in L^{1}((a, b), \mathbb{R})$, with $q^{m}, q_{m}$ defined in (1.15) satisfy (1.18) and $g:[a, b] \times \mathbb{R} \mapsto \mathbb{R}$ be a Carathéodory function such that for a.e. $t \in[a, b]$

$$
\begin{aligned}
& \left(g(t, s)-q^{m}\right) \geq 0 \quad \text { for } s \geq d>0, \\
& \left(g(t, s)-q_{m}\right) \leq 0 \quad \text { for } s \leq-d .
\end{aligned}
$$

Let $h_{0}:[0,+\infty) \mapsto \mathbb{R}$ be a continuous function such that $\lim _{s \rightarrow+\infty} h_{0}(s)=$ $+\infty$, and

$$
g(t, s) \leq h_{0}(s) \quad \forall s \geq d \text {, a.e. } t \in[a, b],
$$

if

$$
\limsup _{s \rightarrow+\infty} T_{h_{0}}(s)>b-a,
$$

then problem (4.1) has at least one solution.

Proof. The proof is based on Lemma 4.2, to this end define

$$
\tilde{q}=\frac{q^{m}+q_{m}}{2}
$$

and set

$$
\tilde{g}(t, s)=g(t, s)-\tilde{q}, \quad q_{0}(t)=q(t)-\tilde{q} .
$$


Now define a continuous function $e: \mathbb{R} \mapsto \mathbb{R}$ such that $e(s) s>0$ for $s \neq 0$. In addition $e$ satisfies

$$
\begin{array}{ll}
e(s) \leq 2\left(q^{m}-\tilde{q}\right)+1 & \forall s \geq 0, \\
e(s)>\left(q^{m}-\tilde{q}\right) & \forall s>d, \\
e(s) \geq-2\left(\tilde{q}-q_{m}\right)-1 & \forall s \leq 0, \\
e(s)<-\left(\tilde{q}-q_{m}\right) & \forall s<-d .
\end{array}
$$

Next define a one-parameter family of functions by

$$
g^{*}(t, s, \lambda):=(1-\lambda) e(s)+\lambda \tilde{g}(t, s) \text { for } \lambda \in[0,1],
$$

so that $g^{*}(t, s, 1)=\tilde{g}(t, s)$ for all $s \in \mathbb{R}$ and a.e. $t \in[a, b]$. Also if

$$
h(s):=h_{0}(s)+|\tilde{q}|+2 \max \left\{q^{m}-\tilde{q}, \tilde{q}-q_{m}\right\}+1,
$$

and taking into account that $\tilde{g}(t, s) \operatorname{sign}(s) \geq 0$ for all $|s|>d$, we obtain that

$$
g^{*}(t, s, \lambda) \leq h(s)
$$

for all $s \geq d$, for a.e. $t \in[a, b]$, and all $\lambda \in[0,1]$.

Next we consider the problem

$$
\begin{gathered}
\left(\phi\left(\frac{u^{\prime}}{\lambda}\right)\right)^{\prime}+g^{*}(t, u, \lambda)=q_{0}(t), \\
u^{\prime}(a)=0, \quad u(\eta)=u(b) .
\end{gathered}
$$

We now show that $g^{*}$ in (4.43) satisfies hypothesis (4.6) of Lemma 4.2.

Set

$$
\left(q_{0}\right)^{m}=\sup _{s \in(a, b]} \frac{1}{s-a} \int_{a}^{s} q_{0}(t) d t, \quad\left(q_{0}\right)_{m}=\inf _{s \in(a, b]} \frac{1}{s-a} \int_{a}^{s} q_{0}(t) d t .
$$

Then,

$$
\left(q_{0}\right)^{m}=q^{m}-\tilde{q} \geq 0, \quad\left(q_{0}\right)_{m}=q_{m}-\tilde{q} \leq 0 .
$$

On the other hand, since

$$
\begin{aligned}
& g^{*}(t, u, \lambda)-\left(q_{0}\right)^{m}=(1-\lambda) e(s)+\lambda\left(g(t, s)-q^{m}\right)+(1-\lambda)\left(\tilde{q}-q^{m}\right), \\
& g^{*}(t, u, \lambda)-\left(q_{0}\right)_{m}=(1-\lambda) e(s)+\lambda\left(g(t, s)-q_{m}\right)+(1-\lambda)\left(\tilde{q}-q_{m}\right),
\end{aligned}
$$

it follows from (4.34) and the definition of $e$ that hypothesis (4.6) of Lemma 4.2 holds, thus the conclusion of that lemma applies to problem (4.43).

Claim 4.4. There are arbitrarily large levels $R$ where the maximum of any solution to (4.43), with $\lambda \in(0,1)$, is not achieved. 
To prove this claim we argue by contradiction. Thus assume that

$$
\max u=R>d
$$

for some solution $u$ of (4.43), with $0<\lambda<1$. By Lemma 4.2, there is a $\tilde{t} \in$ $[a, b]$, such that

$$
-d \leq u(\tilde{t}) \leq d
$$

Thus there exists an interval $\left[t_{1}, t_{2}\right] \subset[a, b]$ such that either $u\left(t_{1}\right)=d, u\left(t_{2}\right)=$ $u_{\max }=R$ and $d<u(t)<R$ for all $t \in\left(t_{1}, t_{2}\right)$, or $u\left(t_{1}\right)=u_{\max }=R, u\left(t_{2}\right)=d$ and $d<u(t)<R$ for all $t \in\left(t_{1}, t_{2}\right)$.

We only consider the first case, the other being the same. Set

$$
Q_{0}(t):=\int_{a}^{t} q_{0}(s) d s, \quad y=\phi\left(\frac{u^{\prime}}{\lambda}\right)-Q_{0}(t),
$$

and rewrite the equation in (4.43) as

$$
u^{\prime}=\lambda \phi^{-1}\left(y+Q_{0}(t)\right), \quad y^{\prime}=-g^{*}(t, u, \lambda) .
$$

We note first that since $u(t) \geq d$ for all $t \in\left[t_{1}, t_{2}\right]$, the condition on $g^{*}$ gives that $y$ is strictly decreasing in $\left[t_{1}, t_{2}\right]$, that is,

$$
y(t) \leq y\left(t_{1}\right) \quad \forall t \in\left[t_{1}, t_{2}\right] .
$$

Therefore,

$$
u^{\prime}(t)=\lambda \phi^{-1}\left(y(t)+Q_{0}(t)\right) \leq \phi^{-1}\left(y\left(t_{1}\right)+\left|Q_{0}\right|_{\infty}\right),
$$

so that

$$
\begin{aligned}
R-d & =u\left(t_{2}\right)-u\left(t_{1}\right)=\int_{t_{1}}^{t_{2}} u^{\prime}(s) d s \\
& \leq \int_{t_{1}}^{t_{2}} \phi^{-1}\left(y\left(t_{1}\right)+\left|Q_{0}\right|_{\infty}\right) d s \\
& \leq(b-a) \phi^{-1}\left(y\left(t_{1}\right)+\left|Q_{0}\right|_{\infty}\right) .
\end{aligned}
$$

From here we conclude that if we choose $R$ sufficiently large, namely

$$
R>\mathscr{D}+d
$$

where $\mathscr{D}:=(b-a) \phi^{-1}\left(2\left|Q_{0}\right|_{\infty}\right)$, then $y\left(t_{1}\right)>\left|Q_{0}\right|_{\infty}$ and thus there exists a unique point $t^{*} \in\left(t_{1}, t_{2}\right)$ such that $y\left(t^{*}\right)=\left|Q_{0}\right|_{\infty}$. Furthermore

$$
u^{\prime}(t)>0, \quad y^{\prime}(t)<0 \quad \text { for a.e. } t \in\left[t_{1}, t^{*}\right],
$$

implying that $y$ is strictly decreasing and $u$ is strictly increasing in $\left[t_{1}, t^{*}\right]$. 
Now we do some estimates for $u\left(t_{2}\right)-u(t), t^{*} \leq t \leq t_{2}$. To this end, we first estimate $u^{\prime}$ in this interval. We have

$$
\begin{aligned}
u^{\prime}(s) & =\lambda \phi^{-1}\left(y(s)+Q_{0}(s)\right) \leq \lambda \phi^{-1}\left(y\left(t^{*}\right)+Q_{0}(s)\right) \\
& =\lambda \phi^{-1}\left(\left|Q_{0}\right|_{\infty}+Q_{0}(s)\right) \leq \phi^{-1}\left(2\left|Q_{0}\right|_{\infty}\right) .
\end{aligned}
$$

Thus, we obtain

$$
\int_{t}^{t_{2}} u^{\prime}(s) d s \leq\left(t_{2}-t\right) \phi^{-1}\left(2\left|Q_{0}\right|_{\infty}\right) \leq \mathscr{D}
$$

and hence

$$
u(t) \geq R-\mathscr{D} \quad \text { for } t \in\left[t^{*}, t_{2}\right] .
$$

Now, from system (4.50) and the definition of $h$ in (4.41), we obtain

$$
h(u(t)) u^{\prime}(t)+\phi^{-1}\left(y(t)-\left|Q_{0}\right|_{\infty}\right) y^{\prime}(t) \geq 0 \quad \text { a.e. on }\left[t_{1}, t^{*}\right],
$$

and thus

$$
\frac{d}{d t}\left[H(u(t))+\Phi^{*}\left(y(t)-\left|Q_{0}\right|_{\infty}\right)\right] \geq 0,
$$

where $H(s)=\int_{0}^{s} h(t) d t$.

Integrating (4.60) from $t$ to $t^{*}$ and recalling that $y\left(t^{*}\right)=\left|Q_{0}\right|_{\infty}$, we find

$$
\Phi^{*}\left(y(t)-\left|Q_{0}\right|_{\infty}\right) \leq H(R)-H(u(t)),
$$

and thus

$$
y(t)+Q_{0}(t) \leq 2\left|Q_{0}\right|_{\infty}+\Psi[H(R)-H(u(t))],
$$

where as before $\Psi$ denotes the right inverse of $\Phi^{*}$. Since $u^{\prime}=\lambda \phi^{-1}\left(y+Q_{0}\right)$, we obtain

$$
\frac{u^{\prime}(t)}{\phi^{-1}\left(2\left|Q_{0}\right|_{\infty}+\Psi[H(R)-H(u(t))]\right)} \leq 1 .
$$

Hence integrating from $t_{1}$ to $t^{*}$,

$$
\int_{t_{1}}^{t^{*}} \frac{u^{\prime}(t) d t}{\phi^{-1}\left(2\left|Q_{0}\right|_{\infty}+\Psi[H(R)-H(u(t))]\right)} \leq t^{*}-t_{1},
$$

and using that $u\left(t^{*}\right) \geq R-\mathscr{D}$, we find that

$$
\int_{d}^{R-\mathscr{D}} \frac{d u}{\phi^{-1}\left(2\left|Q_{0}\right|_{\infty}+\Psi[H(R)-H(u)]\right)} \leq t^{*}-t_{1} .
$$


Now since from [6, Lemma 3.1], it holds that

$$
\begin{gathered}
\int_{0}^{d} \frac{d u}{\phi^{-1}\left(2\left|Q_{0}\right|_{\infty}+\Psi[H(R)-H(u)]\right)} \longrightarrow 0 \quad \text { as } R \longrightarrow+\infty, \\
\int_{R-\mathscr{D}}^{R} \frac{d u}{\phi^{-1}\left(2\left|Q_{0}\right|_{\infty}+\Psi[H(R)-H(u)]\right)} \longrightarrow 0 \quad \text { as } R \longrightarrow+\infty,
\end{gathered}
$$

then from [6, Lemma 3.3], with $K=2\left|Q_{0}\right|_{\infty}$, and (4.65), (4.66), we obtain that

$$
\limsup _{R \rightarrow+\infty} T_{h}(R)=\limsup _{R \rightarrow+\infty} \int_{0}^{R} \frac{d u}{\phi^{-1}\left(\Psi_{\mathrm{r}}(H(R)-H(u))\right)} \leq b-a,
$$

and hence from [6, Lemma 3.4], and (4.41), we finally find that

$$
\limsup _{R \rightarrow+\infty} T_{h_{0}}(R) \leq b-a \text {. }
$$

Since this contradicts (4.36), the claim is proved. The remaining part of the proof is a direct application of Lemma 4.2(iii).

Remark 4.5. Theorem 4.3 was proved under a one-sided growth condition at $+\infty$ for the function $g$. A symmetric result under a growth restriction at $-\infty$ on $g$ can be obtained by applying Theorem 4.3 to problem (4.1) after the change of variables $u \rightarrow-u$.

As in [6] a variant of Theorem 4.3 in which a two-sided condition on $g$ holds is the following.

THEOREM 4.6. Suppose that the odd increasing homeomorphism $\phi$ from $\mathbb{R}$ onto $\mathbb{R}$ satisfies the lower $\sigma$-condition, $q \in L^{1}((a, b), \mathbb{R})$, with $q^{m}, q_{m}$ defined in (1.15) satisfying (1.18), and $g:[a, b] \times \mathbb{R} \mapsto \mathbb{R}$ is a Carathéodory function such that for a.e. $t \in[a, b]$

$$
\begin{aligned}
& \left(g(t, s)-q^{m}\right) \geq 0 \quad \text { for } s \geq d>0, \\
& \left(g(t, s)-q_{m}\right) \leq 0 \quad \text { for } s \leq-d .
\end{aligned}
$$

Let $h_{0}: \mathbb{R} \mapsto \mathbb{R}$ be a continuous function such that $\lim _{s \rightarrow+\infty} h_{0}(s) \operatorname{sign}(s)=$ $+\infty$, and

$$
|g(t, s)| \leq\left|h_{0}(s)\right| \quad \forall|s| \geq d \text {, for a.e. } t \in[a, b] \text {. }
$$

If either

$$
\liminf _{s \rightarrow-\infty} T_{h_{0}}(s)+\limsup _{s \rightarrow+\infty} T_{h_{0}}(s)>b-a
$$


or

$$
\limsup _{s \rightarrow-\infty} T_{h_{0}}(s)+\liminf _{s \rightarrow+\infty} T_{h_{0}}(s)>b-a,
$$

then problem (4.1) has at least one solution.

We omit the proof of this theorem since it is similar to the proof of Theorem 4.3. See also [5] for related results for the case of the linear differential operator and periodic boundary conditions and [6] for the Neumann case and a general operator $\left(\phi\left(u^{\prime}\right)\right)^{\prime}$.

We end this section with the proof of Theorem 1.3.

Proof of Theorem 1.3. We check that the conditions of Theorem 4.3 are satisfied. Clearly conditions (4.34) hold. Now define

$$
h_{0}(s)=g(s)+\varepsilon \phi_{p}(s),
$$

with

$$
0<\varepsilon<\left(\frac{\pi_{p}}{b-a}\right)^{p}-\liminf _{s \rightarrow+\infty} \frac{p G(s)}{|s|^{p}},
$$

and where $G(s):=\int_{0}^{s} g(t) d t$. Since $h_{0}(s) / \phi_{p}(s)>\varepsilon$, for all $s \neq 0$, we have that $h_{0}(s) \rightarrow+\infty$ as $s \rightarrow+\infty$. Thus the proof will be concluded if we show that

$$
\limsup _{s \rightarrow+\infty} T_{h_{0}}(s)>b-a .
$$

Integrating (4.73), and using (4.74), we find that

$$
\liminf _{s \rightarrow+\infty} \frac{p H_{0}(s)}{|s|^{p}}<\left(\frac{\pi_{p}}{b-a}\right)^{p},
$$

where $H_{0}(s)=\int_{0}^{s} h_{0}(t) d t$. Hence from $(v)$ of [16, Corollary 2.6], it follows that (4.75) holds true.

\section{Acknowledgement}

MG-H was supported by a Fondecyt grant 1990428, CG was supported by a Fondecyt grant 7990040, and RM was supported by Fondap-Conicyt.

\section{References}

[1] D. G. de Figueiredo and B. Ruf, On a superlinear Sturm-Liouville equation and a related bouncing problem, J. Reine Angew. Math. 421 (1991), 1-22. MR 92j:34037. Zbl 732.34024.

[2] M. del Pino, M. Elgueta, and R. Manásevich, A homotopic deformation along $p$ of a Leray-Schauder degree result and existence for $\left(\left|u^{\prime}\right|{ }^{p-2} u^{\prime}\right)^{\prime}+f(t, u)=$ $0, u(0)=u(T)=0, p>1$, J. Differential Equations 80 (1989), no. 1, 1-13. MR 91i:34018. Zbl 708.34019. 
212 Solvability for nonlinear three point

[3] W. Feng and J. R. L. Webb, Solvability of three point boundary value problems at resonance, Nonlinear Anal. 30 (1997), no. 6, 3227-3238. MR 99a:34053. Zbl 891.34019.

[4] L. Fernandes and F. Zanolin, Periodic solutions of a second order differential equation with one-sided growth restrictions on the restoring term, Arch. Math. (Basel) 51 (1988), no. 2, 151-163. MR 89i:34053. Zbl 665.34045.

[5] A. Fonda and F. Zanolin, On the use of time-maps for the solvability of nonlinear boundary value problems, Arch. Math. (Basel) 59 (1992), no. 3, 245-259. MR 93i:34035. Zbl 766.34012.

[6] M. García-Huidobro, R. Manásevich, and F. Zanolin, Strongly nonlinear secondorder ODEs with unilateral conditions, Differential Integral Equations 6 (1993), no. 5, 1057-1078. MR 94g:34027. Zbl 785.34023.

[7] A A Fredholm-like result for strongly nonlinear second order ODEs, J. Differential Equations 114 (1994), no. 1, 132-167. MR 95k:34033. Zbl 835.34028.

[8] - Strongly nonlinear second-order ODEs with rapidly growing terms, J. Math. Anal. Appl. 202 (1996), no. 1, 1-26. MR 98b:34022. Zbl 996.47166.

[9] C. P. Gupta, Solvability of a three-point nonlinear boundary value problem for a second order ordinary differential equation, J. Math. Anal. Appl. 168 (1992), no. 2, 540-551. MR 93h:34033. Zbl 763.34009.

[10] _ A note on a second order three-point boundary value problem, J. Math. Anal. Appl. 186 (1994), no. 1, 277-281. MR 95g:34033. Zbl 805.34017.

[11] _ A second order m-point boundary value problem at resonance, Nonlinear Anal. 24 (1995), no. 10, 1483-1489. MR 96e:34036. Zbl 824.34023.

[12] C. P. Gupta, S. K. Ntouyas, and P. Ch. Tsamatos, On an m-point boundary-value problem for second-order ordinary differential equations, Nonlinear Anal. 23 (1994), no. 11, 1427-1436. MR 96j:34023. Zbl 815.34012.

[13] Solvability of an m-point boundary value problem for second order ordinary differential equations, J. Math. Anal. Appl. 189 (1995), no. 2, 575-584. MR 96d:34016. Zbl 819.34012.

[14] R. Manásevich and J. Mawhin, Periodic solutions for nonlinear systems with pLaplacian-like operators, J. Differential Equations 145 (1998), no. 2, 367-393. MR 99c:34034.

[15] R. Manásevich and K. Schmitt, Boundary value problems for quasilinear second order differential equations, Non-Linear Analysis and Boundary Value Problems for Ordinary Differential Equations (Udine), Springer, Vienna, 1996, CISM Courses and Lectures 371 International centre for mechanical sciences, pp. 79119. MR 99a:34059.

[16] R. Manásevich and F. Zanolin, Time-mappings and multiplicity of solutions for the one-dimensional p-Laplacian, Nonlinear Anal. 21 (1993), no. 4, 269-291. MR 94f:34052. Zbl 792.34021.

[17] J. Mawhin, Topological Degree Methods in Nonlinear Boundary Value Problems, Regional Conference Series in Mathematics, vol. 40, American Mathematical Society, Rhode Island, 1979. MR 80c:47055. Zbl 414.34025.

[18] J. Mawhin and J. R. Ward, Periodic solutions of some forced Liénard differential equations at resonance, Arch. Math. (Basel) 41 (1983), no. 4, 337-351. MR 85f:34075. Zbl 537.34037.

[19] P. Omari, G. Villari, and F. Zanolin, Periodic solutions of the Liénard equation with one-sided growth restrictions, J. Differential Equations 67 (1987), no. 2, 278-293. MR 88m:34040. Zbl 615.34037. 
[20] K. Schmitt, Periodic solutions of a forced nonlinear oscillator involving a onesided restoring force, Arch. Math. (Basel) 31 (1978/79), no. 1, 70-73. MR 80a:34045. Zbl 399.34035.

M. García-Huidobro: Departamento de Matemáticas, Pontificia Universidad Católica de Chile, Casilla 306, Correo 22, Santiago, Chile

E-mail address: mgarcia@mat.puc.cl

C. P. Gupta: Department of Mathematics, 084, University of Nevada, Reno, NV 89557, USA

E-mail address: gupta@unr.edu

R. Manásevich: Departamento de Ingeniería Matemática, Universidad de Chile and Centro de Modelamiento Matemático, UMR 2071 CNRS-UChile, Casilla 170, Correo 3, Santiago, Chile

E-mail address: manasevi@dim.uchile.cl 


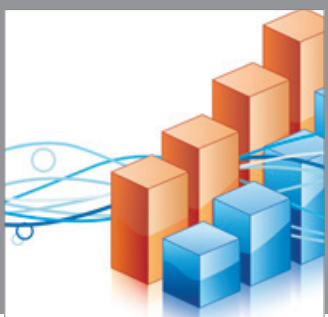

Advances in

Operations Research

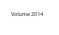

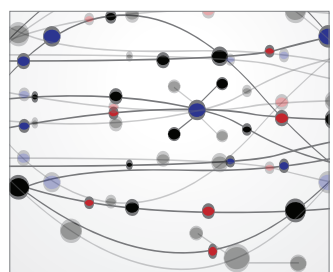

\section{The Scientific} World Journal
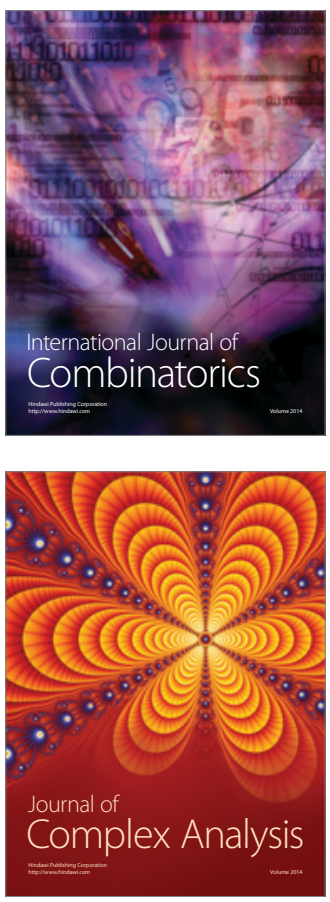

International Journal of

Mathematics and

Mathematical

Sciences
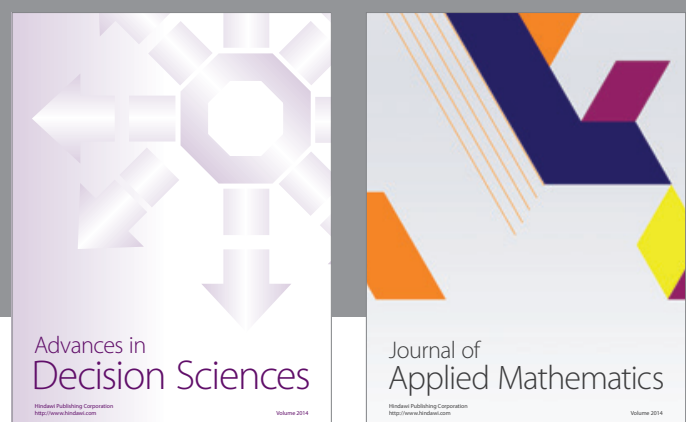

Journal of

Applied Mathematics
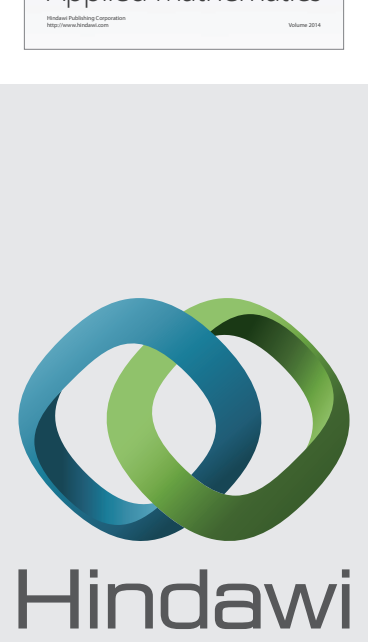

Submit your manuscripts at http://www.hindawi.com
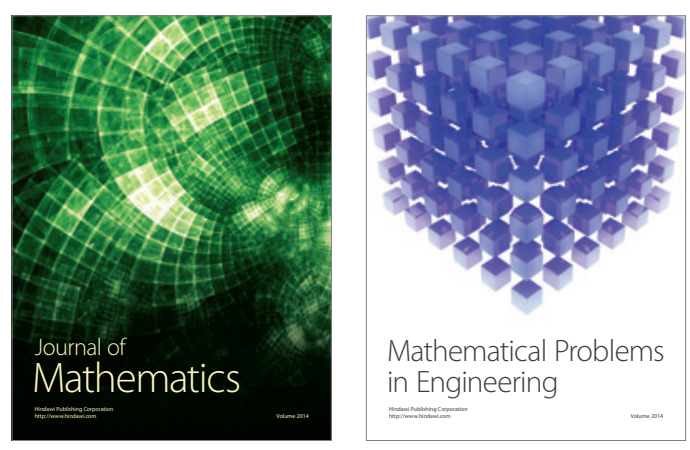

Mathematical Problems in Engineering
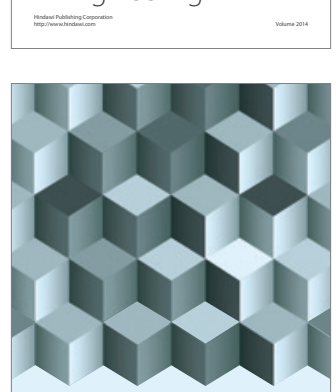

Journal of

Function Spaces
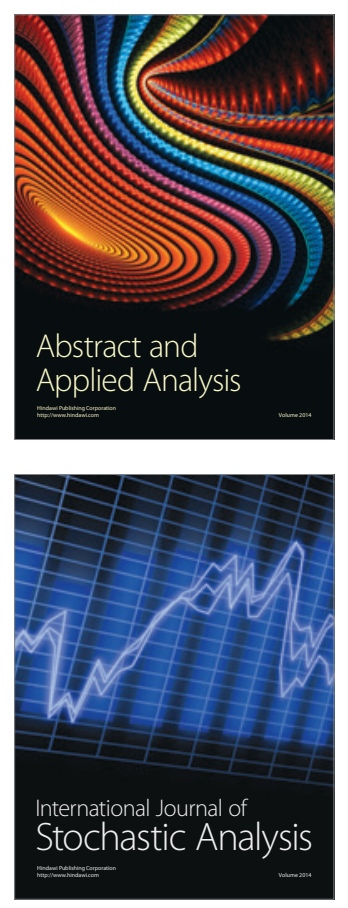

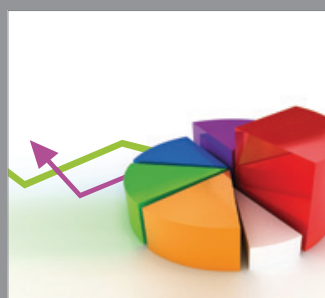

ournal of

Probability and Statistics

Promensencen
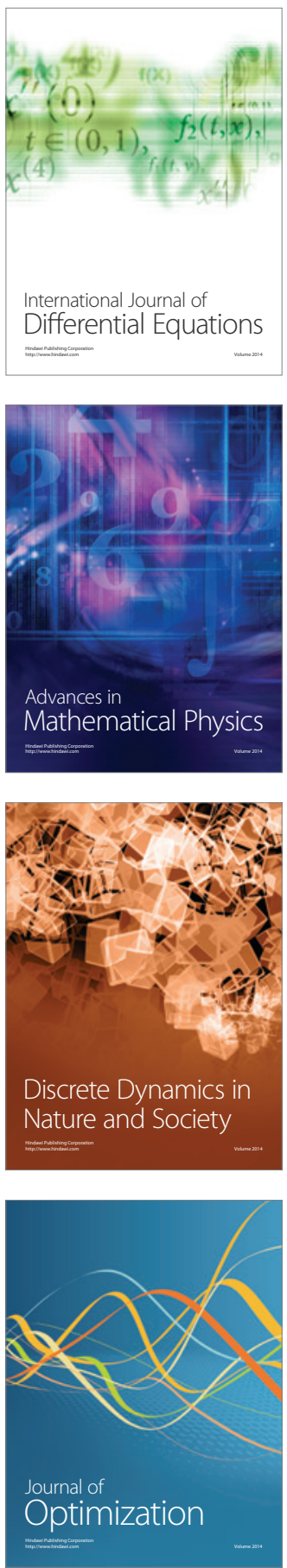